\title{
Variations of porosity in a sheared pressurized layer of saturated soil induced by vertical drainage of water
}

\author{
By FRANCESCO DELL'ISOLA ${ }^{1}$ And Kolumban HutTeR ${ }^{2}$ \\ ${ }^{1}$ Dipartimento di Ingegneria Strutturale e Geotecnica, \\ Università di Roma La Sapienza, Via Eudossiana 18, \\ 00184, Roma, Italy (isola@scilla.ing.uniroma1.it) \\ ${ }^{2}$ Institut für Mechanik, Technische Universität Darmstadt, \\ D-64289 Darmstadt, Germany (hutter@mechanik.tu-darmstadt.de)
}

Received 21 April 1998; revised 21 October 1998; accepted 24 November 1998

\begin{abstract}
Archimedes to Eratosthenes greeting... I. . explain... the peculiarity of a certain method with which furnished you will be able to make a beginning in the investigations by mechanics of some of the problems in mathematics. I am persuaded that this method is no less useful even for the proof of the theorems themselves. For some things first became clear to me by mechanics, though they had later to be proven geometrically...; but it is, of course, easier to provide the proof when some knowledge of the things sought has been acquired by this method rather than to seek it with no prior knowledge. $\dagger$
\end{abstract}

We use the mixture model of soil saturated by a fluid, as developed by dell'Isola \& Hutter and applied to an isothermal steady simple shear flow pressed and drained by a steady flow of water from above. The governing equations are reduced to a single second-order ordinary differential equation (ODE) for the solid-volume fraction; its coefficients depend on the fluid viscosity and the thermodynamic pressure. The coefficients of this ODE give rise to the application of perturbation techniques; the solutions constructed in this way demonstrate that when the thermodynamic pressure is ignored, the solid-volume profile varies unrealistically largely over the layer thickness. Furthermore, when the vertical fluid convective acceleration terms are incorporated, they give rise to a 'destabilizing' mechanism in the sense that a boundary layer over which large changes of the solid-volume fraction arise and which is located where the draining fluid enters may flip to the exit boundary, and so make effective fluidities against shear deformations large. So, depending on the amount of water flow through the layer, the horizontal shearing to prescribed shear tractions may be small or large. For ice-sheet flow situations on soft beds, the flow rates achieving this flip are of the order of a few tens of centimetres per year and are, thus, fairly realistic.

Keywords: drainage-shear-flow interaction; destabilization by drainage water; porosity dependence of viscosities; till behaviour below ice sheets; sliding on soft beds

$\dagger$ Archimedes, The method, Preface Archim.ed. Heiberg ii.426.3-430.22 (translated by Thomas (1941, pp. 220-221)). 


\section{Introduction}

In dell'Isola \& Hutter (1998a), referred to, henceforth, as DH-I, we presented a binary mixture model of a granular solid saturated with a fluid, both components of which are true-density-preserving. In the following soil-mechanics application we mean by fluid the 'pore water' and by solid the 'till'. This model may serve for the description of the dynamics of the thin sediment-water layer below temperate glaciers or ice sheets. In a subsequent paper devoted to a qualitative analysis of the dynamics of a sheared and pressurized layer of saturated soil, we showed how the across-layerdensity distribution could be computed, if (i) the flow was steady and occurring in vertical planes, (ii) all fields except the saturation pressure were assumed to depend on the vertical across-layer coordinate alone, (iii) acceleration terms were ignored, the fluid viscosity was assumed constant, and (iv) isothermal conditions prevailed (see dell'Isola \& Hutter (1998b), henceforth referred to as DH-II). The layer was pressurized from above by an incumbent pressure $p_{\mathrm{i}}$ and sheared by a shear traction $S$; furthermore, a prescribed flow of water from above was assumed, which, together with its equal discharge at the lower boundary, determined the solid-volume fraction profile across the layer.

The model in DH-II is based on a thermodynamic mixture theory of Svendsen \& Hutter (1995). The main feature of this improved theory over common mechanical models of saturated soils consists of the introduction of a 'new' constitutive quantity, which was called thermodynamic or configurational pressure, and in the assumption that the interstitial fluid is viscous. The thermodynamic pressure $\beta_{\mathbf{s}}(\nu, \vartheta)$ is a function of the solid-volume fraction $\nu$ and the temperature $\vartheta$, and is obtained from the inner free (Helmholtz) energy $\psi_{\mathrm{I}}(\nu, \vartheta)$ via

$$
\rho \frac{\partial \psi_{\mathrm{I}}(\nu, \vartheta)}{\partial \nu}=\beta_{\mathrm{s}}(\nu, \vartheta),
$$

in which $\rho$ denotes the mixture density. Equation (1.1) expresses the well-known thermodynamic relation between the free energy and the stress. Its physical meaning seems clear: the pressure $\beta_{\mathrm{s}}$ results from the 'strain' induced by the redistribution of the pores; a part of the stress arising in a solid-fluid mixture depends on this kinematic variable $\nu$ in a way that is dictated by the corresponding dependence of the free energy.

Another variable, called saturation pressure $p$, also enters this model. It represents the constraint force to the kinematic constraint that the fluid fills the entire pore space. Clearly, if $\nu_{\mathrm{f}}$ and $\nu_{\mathrm{s}}$ are the volume fractions of the fluid and the solid, respectively, then the condition of saturation means that $\nu_{\mathrm{f}}+\nu_{\mathrm{s}}=1$; so the two volume fractions are no longer independent and the loss of a field variable must be compensated for by a constraint field, the saturation pressure $p$. Thus, two pressures characterize this model. A special reduction can, however, be brought into coincidence with some of the classical soil-mechanics literature; assume, for instance, that the inner free energy does not depend on $\nu_{\mathrm{f}}=\nu$. Then the thermodynamic pressure vanishes and only $p$ survives. Hutter et al. (1994) proved that gravity shear flow down an inclined plane of a certain binary viscous mixture admits no solution in this case. So, what is classical and used by some soil mechanicians appears to be dubious in the till-layer context. Therefore, an extension of the model equations was needed. The model equations in DH-I and DH-II achieve this via the thermodynamic 
pressure $\beta_{\mathrm{s}}$, which, by the Viennese experts of the early 20th century (Terzaghi and Fillunger - see de Boer \& Ehlers (1990)), was associated with effective stress, and $\beta_{\mathrm{s}}$ essentially plays that role, but we refrain from calling it such.

In more traditional approaches to soil mechanics, the two pressures are sometimes not differentiated or $\beta_{\mathrm{s}}$ is simply dropped, and the remaining pressure identified with the effective pressure of Terzaghi. In the Boulton \& Hindmarsh (1987) and Clarke (1987) approaches, what is called effective pressure appears to agree with what we call saturation pressure, $p$, but these authors do not recognize its constraint role and use it as an independent constitutive variable for the viscosity. Such a constitutive dependence is not possible for a constraint variable. We therefore introduce $\beta_{\mathrm{s}}$ and choose it via (1.1) to be functionally dependent on the solid-volume fraction, as we do with the viscosity. In a way, we and Boulton \& Hindmarsh (1987) are attempting analogous things with inverse roles of some dependent and independent constitutive variables.

It was made clear in DH-I that a mixture theory of the complexity of that of Svendsen \& Hutter (1995) is needed if the thermodynamic processes arising in till layers below ice sheets are likely to be realistically modelled. The theory also has its merits on the level of its restriction to purely mechanical processes, however. Indeed, it was shown in DH-II that in a simple steady shear flow of a layer of sedimentwater, in which water is pressed through the layer and all variables vary only with the across-layer coordinate, that the solid-volume fraction profile is governed by a secondorder ordinary differential equation (ODE) of which the coefficient functions depend on the thermodynamic pressure and the fluid viscosity. Without these constitutive quantities (which, incidentally, are missing in some classical theories), the variation of the solid-volume fraction across the layer cannot even be determined.

In DH-II, it was further demonstrated that for Stokes flow, i.e. when the acceleration terms for both the solid and the fluid are ignored, the constitutive relation for $\beta_{\mathrm{s}}$ is crucial in characterizing drainage phenomena in granular solids. In particular, when the convective time rate of the fluid flow can be neglected, a thermodynamic stability condition (derived in DH-I) implies very stringent restrictions on the constitutive relation for $\beta_{\mathrm{s}}$ : these restrictions have, as a consequence, the localization of a sharp boundary layer for the solid-volume fraction profiles at the boundary of the mixture at which the fluid is flowing in. Furthermore, if a constant fluid viscosity was assumed, it could be shown that analytically determined solid-volume fraction profiles turned out to be unphysical, because $\nu$ was negative or greater than unity in some regions of the layer (dell'Isola \& Hutter 1999, henceforth referred to as DH-III).

The above results are inherently connected with the thermodynamics of Svendsen \& Hutter (1995) and are as applied to this till-layer situation in DH-I, DH-II and DHIII. The boundary layer, if at the top, has large porosity compared to the remaining layer; if it is at the bottom then the reverse applies. Thus, supposing a dependence of the solid (and fluid) viscosities upon porosity such that high porosity induces a viscosity decrease, we anticipate a large resistance of the till layer against horizontal shear when the boundary layer is at the top, and a small one when it is at the bottom.

In the present paper, developing the ideas introduced in DH-I, DH-II and DH-III and limiting ourselves to the case in which all considered fields depend only on a vertical coordinate $z$, we

1. study the vertical-stationary fluid drainage through porous pressurized solids and its influence on the horizontal-stationary shear flow when the vertical fluid 
convective time rate of change and the dependence of the fluid viscosity on the solid-volume fraction cannot be neglected;

2. introduce a dimensionless form of the ODE for the solid-volume fraction $\nu$ alone;

3. introduce, in terms of the relevant constitutive and kinematic quantities, the dimensionless numbers that determine, quantitatively and qualitatively, the properties of the reduced ODE (and, therefore, the behaviour of the considered systems);

4. prove (using the arguments from Bush (1992) as adapted in DH-II) that there exists a transcendental localizing functional controlling the localization of the boundary layer of solid-volume fraction: when this function is negative (positive) for all values attained by the solid-volume fraction, the boundary layer is located at that part of the boundary into (out of) which the fluid is flowing in (out);

5. apply the model to the study of the drainage through till-pore water layers and prove (using the estimates proposed in DH-I and DH-II) that ice-melting rates of a few tens of centimetres per year can cause a flip from the top to the bottom boundary.

We explicitly remark that we have proved that

(i) the model for the till-pore water layer below ice sheets may predict the existence of destabilizing mechanisms under simple shear, thus theoretically substantiating the results and conjectures found in the literature (see, for example, Alley et al. 1987a,b; MacAyeal 1992);

(ii) these mechanisms are controlled by the relative order of magnitude of the fluid vertical convective time rate and the thermodynamic quantities $F(\nu) / \nu$ defined in formula (2.8) and the fluid viscosity $\mu_{\mathrm{f}}(\nu)$; and

(iii) the threshold for ice melting rate, predicted in the proposed model over which the boundary-layer flip may be initiated, is sufficiently low that a collapse of an ice sheet cannot be ruled out.

\section{Equation for the solid-volume fraction profile in a drained pressurized granular solid}

Consider a layer of a saturated sediment-water mixture bounded above by ice (and water) and below by a rock bed. The moving ice exerts a pressure and shear traction at the upper boundary and drives a mass flow of water across the interface. For the purposes of this paper, these quantities are assumed to be prescribed. The water entering the layer is drained at the lower interface into the rock bed; it simultaneously influences the distribution of the solid-volume fraction and, through the latter, also affects the effective viscosity against shearing, making it smaller when the solidvolume fraction decreases.

Let $\mathrm{O} x z$ be a plane Cartesian coordinate system with origin $\mathrm{O}$, horizontal axis $x$ and vertical axis $z$ pointing upwards opposite to the direction of gravity. Consider 
plane flow, and let the layer of sediment-water mixture be confined to $z \in\left[f_{\mathrm{b}}, h\right]$, where $z=h(x, t)$ and $z=f_{\mathrm{b}}(x, t)$ denote the upper and lower interface surfaces, respectively. In this section, our intention is to derive the ODE for the solid-volume fraction for the simplified case that all considered fields, except the pressure, depend only on the vertical coordinate $z \in\left[f_{\mathrm{b}}, h\right], H=h-f_{\mathrm{b}}$, while the pressure is a linear function of $x$. Moreover, stationary flow conditions will be imposed so that both $f_{\mathrm{b}}$ and $h$ are time independent and can be identified with $z=0$ and $z=H$. Our starting point will be the balance equations of mass and linear momentum for the water and sediment constituents, as derived in DH-II.

(a) Balances of mass and momentum in one-dimensional flows

Let us introduce the set of fields and constants as follows.

$\nu$

solid-volume fraction

$u_{\mathrm{s}}, u_{\mathrm{f}} \quad$ horizontal velocity components of the solid and fluid, respectively

$w_{\mathrm{s}}, w_{\mathrm{f}} \quad$ vertical velocity components of the solid and fluid, respectively

$p \quad$ saturation pressure

$\beta_{\mathrm{s}}(\nu) \quad$ thermodynamic pressure

$\hat{\rho}_{\mathrm{f}}, \hat{\rho}_{\mathrm{s}} \quad$ true fluid and sediment mass densities

$\mu_{\mathrm{f}}, \mu_{\mathrm{s}} \quad$ (apparent) fluid and solid viscosities

$$
\begin{aligned}
& \tilde{\alpha}=: \hat{\rho}_{\mathrm{f}} g / K \\
& a:=\hat{\rho}_{\mathrm{f}} / \hat{\rho}_{\mathrm{s}} \\
& \xi_{\mathrm{s}}:=\rho_{\mathrm{s}} / \rho=\left[\nu \hat{\rho}_{\mathrm{s}} /\left(\nu \hat{\rho}_{\mathrm{s}}+(1-\nu) \hat{\rho}_{\mathrm{f}}\right)\right]=[\nu /(\nu+(1-\nu) a)]
\end{aligned}
$$

$g \quad$ gravity constant

$K \quad$ soil permeability

$\partial p / \partial x \quad$ driving horizontal pressure gradient

(independent of $x$, horizontal coordinate)

For stationary flows, the balance of the mass and the vertical and horizontal projections of the balance of linear momentum lead to

$$
\left.\begin{array}{c}
\left(\nu w_{\mathrm{s}}\right)^{\prime}=0, \\
\left((1-\nu) w_{\mathrm{f}}\right)^{\prime}=0, \\
\left(-\nu \beta_{\mathrm{s}}-\nu p+\mu_{\mathrm{s}} w_{\mathrm{s}}^{\prime}\right)^{\prime}+\left(p+\left(1-\xi_{\mathrm{s}}\right) \beta_{\mathrm{s}}\right) \nu^{\prime}-\nu(1-\nu) \tilde{\alpha}\left(w_{\mathrm{s}}-w_{\mathrm{f}}\right)=\hat{\rho}_{\mathrm{s}} \nu w_{\mathrm{s}} w_{\mathrm{s}}^{\prime}, \\
\left(-(1-\nu) p+\frac{4}{3} \mu_{\mathrm{f}} w_{\mathrm{f}}^{\prime}\right)^{\prime}-\left(p+\left(1-\xi_{\mathrm{s}}\right) \beta_{\mathrm{s}}\right) \nu^{\prime}+\nu(1-\nu) \tilde{\alpha}\left(w_{\mathrm{s}}-w_{\mathrm{f}}\right)=\hat{\rho}_{\mathrm{f}}(1-\nu) w_{\mathrm{f}} w_{\mathrm{f}}^{\prime}, \\
-\nu(\partial p / \partial x)+\left(\mu_{\mathrm{s}} u_{\mathrm{s}}^{\prime}\right)^{\prime}-\nu(1-\nu) \tilde{\alpha}\left(u_{\mathrm{s}}-u_{\mathrm{f}}\right)=\hat{\rho}_{\mathrm{s}} \nu w_{\mathrm{s}} u_{\mathrm{s}}^{\prime}, \\
-(1-\nu)(\partial p / \partial x)+\left(\mu_{\mathrm{f}} u_{\mathrm{f}}^{\prime}\right)^{\prime}+\nu(1-\nu) \tilde{\alpha}\left(u_{\mathrm{s}}-u_{\mathrm{f}}\right)=\hat{\rho}_{\mathrm{f}}(1-\nu) w_{\mathrm{f}} u_{\mathrm{f}}^{\prime},
\end{array}\right\}
$$

in $z \in\left(f_{\mathrm{b}}, h\right), t>0$. The first two equations are mass balances for the solid and fluid, the next two are vertical and the last two horizontal momentum balances. The prime denotes differentiation with respect to $z$, and the thermodynamic pressure $\beta_{\mathrm{s}}(\nu)$ is related to the inner free energy $\psi_{\mathrm{I}}(\nu, \vartheta=$ const. $)$ via

$$
\beta_{\mathrm{s}}(\nu)=\left(\nu \hat{\rho}_{\mathrm{s}}+(1-\nu) \hat{\rho}_{\mathrm{f}}\right)\left(\partial \psi_{\mathrm{I}} / \partial \nu\right) \quad(\nu, \vartheta=\text { const. }) .
$$


These equations correspond to equations (DH-II, (2.1)); as they stand in (2.1) they are partly less general than (DH-II, (2.1)), since they are restricted to steady state, but they are also more general, because they involve, on the right-hand side of the momentum equations, the vertical and horizontal convective acceleration terms, which are quadratic in the velocity components. These non-Stokesian terms, thought insignificant in DH-II, turned out to be important, as will be demonstrated below. The viscosities $\mu_{\mathrm{f}}$ and $\mu_{\mathrm{s}}$ and the free energy $\psi_{\mathrm{I}}$ and $\tilde{\alpha}$ are thought to be functions of the solid-volume fraction, and $\mu_{\mathrm{s}}$ may also depend on the solid stretching tensor invariants, but, for the purposes of this paper, such a dependence is not explicitly used. We also employ isothermal conditions.

The above equations form six ODEs for the unknowns $\nu, w_{\mathrm{s}}, w_{\mathrm{f}}, p, u_{\mathrm{s}}$ and $u_{\mathrm{f}}$. They must be subjected to boundary conditions. For the general situation, these are posed in DH-I and DH-II. In this paper, we restrict considerations to no abrasion of till from the rock bed, $w_{\mathrm{s}}(0)=0$, and to the prescription of the inflow of water from above, $\left|V_{\mathrm{f} 0}\right|$. From the boundary conditions (2.6) and (2.9)-(2.13) in DH-II, we may then deduce the relations

$$
\begin{array}{cc}
\left(\mathfrak{m}_{\mathrm{f}}^{\mathrm{b}} / \hat{\rho}_{\mathrm{f}}\right)\left[-(1-\nu)\left(\nu \beta_{\mathrm{s}}-\mu_{\mathrm{s}} w_{\mathrm{s}}^{\prime}-\frac{4}{3} \mu_{\mathrm{f}} w_{\mathrm{f}}^{\prime}-p_{\mathrm{i}}\right)-\frac{4}{3} \mu_{\mathrm{f}} w_{\mathrm{f}}^{\prime}\right]^{4}=-(1-\nu) w_{\mathrm{f}}, & \text { at } z=0, \\
\left(\nu-\alpha \nu^{l}\right) p_{\mathrm{i}}+\nu(1-\nu) \beta_{\mathrm{s}}-(1-\nu) \mu_{\mathrm{s}} w_{\mathrm{s}}^{\prime}+\frac{4}{3} \nu \mu_{\mathrm{f}} w_{\mathrm{f}}^{\prime}=0, & \text { at } z=H .
\end{array}
$$

Here, $\alpha$ and $l$ are a coefficient and an exponent determining how much of the incumbent pressure $p_{\mathrm{i}}$ is distributed among the constituents $\left(\alpha \nu^{l} p_{\mathrm{i}}\right.$ is carried by the solid), and $\mathfrak{m}_{\mathrm{f}}^{\mathrm{b}}$ is a coefficient of the drainage function, and the latter itself is assumed to be proportional to the fourth power of the fluid pressure acting on the interface.

\section{(b) The ODE for the solid-volume fraction}

As we will assume that there is no addition of solids at the bottom and/or top interface and that there is an inflow $\left|V_{\mathrm{f} 0}\right|$ of fluid in the negative $z$-direction at the top interface, then $(2.1)_{1,2}$ imply

$$
w_{\mathrm{s}}(z, t)=0, \quad w_{\mathrm{f}}(z, t)=-\left[\left|V_{\mathrm{fo}}\right| /(1-\nu(z, t))\right] .
$$

Adding $(2.1)_{3}$ to $(2.1)_{4}$, we obtain

$$
p^{\prime}=\left(-\nu \beta_{\mathrm{s}}+\frac{4}{3} \mu_{\mathrm{f}} w_{\mathrm{f}}^{\prime}\right)^{\prime}-\hat{\rho}_{\mathrm{f}}(1-\nu) w_{\mathrm{f}} w_{\mathrm{f}}^{\prime} .
$$

Introducing this last expression into $(2.1)_{3}$, we finally obtain

$$
\left(\frac{4}{3} \mu_{\mathrm{f}}\left|V_{\mathrm{fo}}\right|\left(\frac{1}{1-\nu}\right)^{\prime}\right)^{\prime}+\hat{\rho}_{\mathrm{f}}\left|V_{\mathrm{f} 0}\right|^{2}\left(\frac{1}{1-\nu}\right)^{\prime}+\frac{F(\nu)}{\nu} \nu^{\prime}-\left|V_{\mathrm{f} 0}\right| \tilde{\alpha}=0,
$$

in which

$$
\frac{F(\nu)}{\nu}:=-(1-\nu)\left(\beta_{\mathrm{s}} \frac{1}{\nu+b}+\frac{\mathrm{d}}{\mathrm{d} \nu} \beta_{\mathrm{s}}\right)
$$

Equation (2.7) is an ordinary nonlinear differential equation in terms of the unknown field $\nu$ alone. It reduces the system (2.1) to a single second-order ODE for the solidvolume fraction profile of which the integration requires two boundary conditions. 
In order to find a more convenient form for (2.7), we introduce the following change of variables

$$
y=\frac{1}{1-\nu} \quad \Rightarrow \quad y^{\prime}=\left(\frac{1}{1-\nu}\right)^{2} \nu^{\prime}
$$

so that (2.7) becomes

$$
\left(\frac{4}{3} \mu_{\mathrm{f}}(\nu)\left|V_{\mathrm{f} 0}\right| y^{\prime}\right)^{\prime}+\left(\hat{\rho}_{\mathrm{f}}\left|V_{\mathrm{f} 0}\right|^{2}+h(\nu(y))\right) y^{\prime}-\left|V_{\mathrm{f} 0}\right| \tilde{\alpha}=0,
$$

where

$$
h(\nu):=-(1-\nu)^{3}\left(\beta_{\mathrm{s}} \frac{1}{\nu+b}+\frac{\mathrm{d} \beta_{\mathrm{s}}}{\mathrm{d} \nu}\right), \quad b=\frac{a}{1-a},
$$

and $\nu$ is understood to be expressed in terms of $y$ through (2.9). Except for the terms involving $\left|V_{\mathrm{fo}}\right|^{2}$, equation (2.10) agrees with equation (3.3) in DH-II. Thus it is this quadratic term that is due to the convective fluid acceleration.

Introducing the scalings

$$
z=[H] \tilde{z}, \quad \mu_{\mathrm{f}}=\left[\mu_{\mathrm{f}}\right] \tilde{\mu}_{\mathrm{f}}, \quad \beta_{\mathrm{s}}(\nu)=\left[\beta_{1}\right] \tilde{\beta}_{\mathrm{s}}(\nu),
$$

in which the bracketed terms have fixed values of a typical order of magnitude for the variable that they are scaling, and the variables $\tilde{\cdot}$ are dimensionless, equation $(2.10)$ can be transformed to the following dimensionless ODE

$$
\frac{\mathrm{d}}{\mathrm{d} \tilde{z}}\left(h_{0} \tilde{\mu}_{\mathrm{f}}(\nu) \frac{\mathrm{d} y}{\mathrm{~d} \tilde{z}}\right)+\mathfrak{d}(\nu(y)) \frac{\mathrm{d} y}{\mathrm{~d} \tilde{z}}-h_{0} h_{2}=0,
$$

where

$$
\mathfrak{d}(\nu):=-(1-\nu)^{3}\left(\tilde{\beta}_{\mathrm{s}} \frac{1}{\nu+b}+\frac{\mathrm{d} \tilde{\beta}_{\mathrm{s}}}{\mathrm{d} \nu}\right)+h_{1}=h(\nu)+h_{1},
$$

and the dimensionless numbers

$$
h_{0}:=\frac{4}{3} \frac{\left[\mu_{\mathrm{f}}\right]\left|V_{\mathrm{f} 0}\right|}{[H]\left[\beta_{1}\right]}, \quad h_{1}:=\frac{\hat{\rho}_{\mathrm{f}}\left|V_{\mathrm{f} 0}\right|^{2}}{\left[\beta_{1}\right]}, \quad h_{2}:=\frac{3}{4} \frac{[H]^{2} \tilde{\alpha}}{\left[\mu_{\mathrm{f}}\right]},
$$

have been introduced. Simple manipulations lead to the following expression for $(2.13)$,

$$
h_{0} \tilde{\mu}_{\mathrm{f}}(\nu) \frac{\mathrm{d}^{2} y}{\mathrm{~d} \tilde{z}^{2}}+\mathfrak{h}(\cdot, \tilde{z}) \frac{\mathrm{d} y}{\mathrm{~d} \tilde{z}}-h_{0} h_{2}=0
$$

in which the functional

$$
\begin{aligned}
\mathfrak{h}(\cdot, \tilde{z}) & :=h_{0} \frac{\mathrm{d} y}{\mathrm{~d} \tilde{z}} \frac{\mathrm{d} \tilde{\mu}_{\mathrm{f}}}{\mathrm{d} y}-(1-\nu)^{3}\left(\tilde{\beta}_{\mathrm{s}} \frac{1}{\nu+b}+\frac{\mathrm{d} \tilde{\beta}_{\mathrm{s}}}{\mathrm{d} \nu}\right)+h_{1} \\
& =h_{0} \frac{\mathrm{d} y}{\mathrm{~d} \tilde{z}} \frac{\mathrm{d} \tilde{\mu}_{\mathrm{f}}}{\mathrm{d} y}+h(\nu)+h_{1}=h_{0} \frac{\mathrm{d} y}{\mathrm{~d} \tilde{z}} \frac{\mathrm{d} \tilde{\mu}_{\mathrm{f}}}{\mathrm{d} y}+\mathfrak{d}(\nu),
\end{aligned}
$$

was introduced. Notice that (2.13) remains invariant when the signs of $h_{0}$ and $\tilde{z}$ change, implying that the profiles for inflow from above and below are mirror pictures 
of one another, as expected. Notice, moreover, that whenever there is no water inflow, $\left|V_{\mathrm{f} 0}\right|=0$, then $h_{0}=0, h_{1}=0$, and the second-order ODE (2.13) reduces to first order,

$$
h(\nu(y))(\mathrm{d} y / \mathrm{d} \tilde{z})=0,
$$

with the solutions $h(\nu(y))=0$ or $\mathrm{d} y / \mathrm{d} \tilde{z}=0$. In both cases, $\nu$ must be constant, a result that could also be obtained by integrating $(2.1)_{2,3}$ directly. Similarly, when $\left|V_{\mathrm{fo}}\right| \neq 0$ but the fluid viscosity is ignored, then $\dagger$

$$
\mathfrak{d}(\cdot, \tilde{z}) \frac{\mathrm{d} y}{\mathrm{~d} \tilde{z}}=\left(h(\nu(y))+h_{1}\right) \frac{\mathrm{d} y}{\mathrm{~d} \tilde{z}}=h_{0} h_{2} ;
$$

for $h_{1}=0$ this equation was treated in detail in DH-II. The general case depends on three dimensionless numbers, $h_{0}, h_{1}$ and $h_{2}$, for which various asymptotic limits can be considered.

The functional $\mathfrak{h}(\cdot, \tilde{z})$ maps the solid-volume fraction profile into a function of the variable $\tilde{z}$ : it is well known (see, for example, Bush 1992; DH-II) that the sign of $\mathfrak{h}$ as a function of $\tilde{z}$ determines the location of the boundary layers (eventually) arising in the solutions of (2.16).

For the ODE (2.13), a first integral can easily be obtained and, for example, can be expressed in the following three equivalent forms:

$$
\left.\begin{array}{r}
h_{0} \tilde{\mu}_{\mathrm{f}}(\nu(y)) \frac{\mathrm{d} y}{\mathrm{~d} \tilde{z}}=-\int_{y(0)}^{y} \mathfrak{d}\left(y_{1}\right) \mathrm{d} y_{1}+h_{0} h_{2} \tilde{z}+h_{0} \tilde{\mu}_{\mathrm{f}}(y(0)) \frac{\mathrm{d} y}{\mathrm{~d} \tilde{z}}(0), \\
h_{0} \tilde{\mu}_{\mathrm{f}}(\nu(y)) \frac{\mathrm{d} y}{\mathrm{~d} \tilde{z}}=-\int_{y(1)}^{y} \mathfrak{d}\left(y_{1}\right) \mathrm{d} y_{1}+h_{0} h_{2}(\tilde{z}-1)+h_{0} \tilde{\mu}_{\mathrm{f}}(y(1)) \frac{\mathrm{d} y}{\mathrm{~d} \tilde{z}}(y(1)), \\
2 h_{0} \tilde{\mu}_{\mathrm{f}}(\nu(y)) \frac{\mathrm{d} y}{\mathrm{~d} \tilde{z}}=-\int_{y(1)}^{y} \mathfrak{d}\left(y_{1}\right) \mathrm{d} y_{1}-\int_{y(0)}^{y} \mathfrak{d}\left(y_{1}\right) \mathrm{d} y_{1}+h_{0} h_{2}(2 \tilde{z}-1) \\
+h_{0} \tilde{\mu}_{\mathrm{f}}(y(1)) \frac{\mathrm{d} y}{\mathrm{~d} \tilde{z}}(y(1))+h_{0} \tilde{\mu}_{\mathrm{f}}(y(0)) \frac{\mathrm{d} y}{\mathrm{~d} \tilde{z}}(0) .
\end{array}\right\}
$$

As a consequence, the functional (2.17) takes the form

$$
\begin{aligned}
\mathfrak{h}(\cdot, \tilde{z}):=\frac{1}{2} \frac{\mathrm{d} \ln \tilde{\mu}_{\mathrm{f}}}{\mathrm{d} y} & \left(-\int_{y(1)}^{y} \mathfrak{d}\left(y_{1}\right) \mathrm{d} y_{1}-\int_{y(0)}^{y} \mathfrak{d}\left(y_{1}\right) \mathrm{d} y_{1}+h_{0} h_{2}(2 \tilde{z}-1)\right. \\
& \left.+\left(h_{0} \tilde{\mu}_{\mathrm{f}}(y(1)) \frac{\mathrm{d} y}{\mathrm{~d} \tilde{z}}(y(1))+h_{0} \tilde{\mu}_{\mathrm{f}}(y(0)) \frac{\mathrm{d} y}{\mathrm{~d} \tilde{z}}(y(0))\right)\right)+\mathfrak{d}(\nu(y)) .
\end{aligned}
$$

The dimensionless quantities $h_{0}, h_{1}$ and $h_{2}$ are exclusively determined by the scales and by $\tilde{\alpha}$. When applying the present model to the drainage phenomenon in the till layer below large ice sheets, as suggested in DH-I and DH-II, the estimates,

$$
\left.\begin{array}{lc}
h_{0} \in\left[2 \times 10^{-21}, 2 \times 10^{-15}\right], & h_{1} \in\left[10^{-24}, 10^{-14}\right], \\
h_{2} \in\left[3.75 \times 10^{13}, 3.75 \times 10^{15}\right], & h_{0} h_{2} \in\left[7.5 \times 10^{-8}, 7.5 \times 10^{0}\right],
\end{array}\right\}
$$

can be obtained by using the values in table 1 . Therefore, the coefficient of the second-order derivative in (2.16) is small so that the differential equation defines a singular perturbation problem.

$\dagger$ The constant $h_{0} h_{2}$ was called $\eta_{\mathrm{f}}$ in DH-II. 
Table 1. Characteristic values of physical quantities

\begin{tabular}{ccc}
\hline variable & value & dimension \\
\hline$\left[\mu_{\mathrm{f}}\right]$ & $2 \times 10^{-3}$ & $\mathrm{~kg} \mathrm{~m}^{-1} \mathrm{~s}^{-1}$ \\
{$\left[V_{\mathrm{f} 0}\right]$} & $3 \times 10^{-3}-3 \times 10^{0}$ & $\mathrm{~m} \mathrm{a}^{-1}$ \\
{$\left[V_{\mathrm{f} 0}\right]$} & $10^{-11}-10^{-8}$ & $\mathrm{~m} \mathrm{~s}^{-1}$ \\
{$[H]$} & $1-10$ & $\mathrm{~m}$ \\
{$\left[\beta_{1}\right]$} & $10^{5}-10^{7}$ & $\mathrm{~Pa}$ \\
$\tilde{\alpha}$ & $10^{9}-10^{11}$ & $\mathrm{~kg} \mathrm{~m}^{-3} \mathrm{~s}^{-1}$ \\
$\hat{\rho}_{\mathrm{f}}$ & $10^{3}$ & $\mathrm{~kg} \mathrm{~m}^{-3}$ \\
$\nu_{\max }$ & $\simeq 0.85$ & - \\
\hline
\end{tabular}

A particular case of this singular perturbation problem was studied in DH-II, where it was proven that if $h_{1}$ and $\partial \tilde{\mu}_{\mathrm{f}} / \partial \nu$ are negligible, then the solid mass fraction is, in the draining layer, nearly constant and equal to its maximum value.

The outer solution $y_{0}(\tilde{z})$ for $(2.16)$ is the solution of the following ODE

$$
\mathfrak{h}(\nu(y)) \frac{\mathrm{d} y}{\mathrm{~d} \tilde{z}}-h_{0} h_{2}=0,
$$

which implies that, when $\tilde{z}$ is outside the arising boundary layer,

$$
y_{0}(\tilde{z}) \simeq y_{\mathrm{e}}, \quad \frac{\mathrm{d} y_{0}}{\mathrm{~d} \tilde{z}}(\tilde{z}) \simeq \frac{h_{0} h_{2}}{\mathfrak{h}\left(\cdot, \tilde{z}\left(y_{\mathrm{e}}\right)\right)},
$$

in which $y_{\mathrm{e}}$ denotes the value of $y$ at the boundary where the boundary layer does not arise. This outer solution formally differs from the zero-fluid-viscosity solution only by replacing $\mathfrak{d}(\nu(y))$ by $\mathfrak{h}(\cdot, \tilde{z})$.

In Appendix A we construct the inner solution for the two cases: that the boundary layer arises at the top and bottom, respectively.

\section{Localization of the boundary layers as determined by $h_{0}, h_{1}$ and the boundary conditions}

\section{(a) Localization of boundary layers}

It follows from the analysis in DH-II that the boundary layer is at the top (bottom) interface, when $\mathfrak{h}$ is negative (positive) in $\tilde{z} \in[0,1]$, whereas when $\mathfrak{h}$ may change sign at $\tilde{z}^{*} \in(0,1)$, such a boundary layer arises at $\tilde{z}=\tilde{z}^{*}$ (and possibly at the interfaces).

The form of the $\mathfrak{h}$-functional in $(2.17)_{1}$ thus lends itself interesting qualitative considerations about the solid-volume fraction profiles as follows.

(i) Note that the second term on the right-hand side of (2.17) is always negative. For sufficiently small $h_{1}$, it then follows that the boundary layer is at the top. Indeed, if it is assumed to be at the top, then, since $\mathrm{d} \tilde{\mu}_{\mathrm{f}} / \mathrm{d} y>0, \mathrm{~d} y / \mathrm{d} \tilde{z}$ will in this case be negative, so that the first term of the right-hand side of (2.17) is also negative, making $\mathfrak{h}$ negative for all $\tilde{z} \in[0,1]$. Note that to make this argument valid, the third term in (2.17) need not necessarily be smaller than the modulus of the second; it suffices if the sum of all three terms is negative. As a consequence, for sufficiently small $h_{1}$, i.e. small convective velocities $w_{\mathrm{f}}$, 
the solid-volume fraction profile outside the boundary layer is almost uniform with a value nearly equal to its (highest-possible) value assumed at the bottom interface.

(ii) On the other hand, for sufficiently large and positive $h_{1}$, the boundary layer is located at the bottom interface: this means that the field $\nu$ assumes the smallest possible value in the greatest part of the drainage layer.

(iii) Alternatively, as $\mathrm{d} \tilde{\mu}_{\mathrm{f}} / \mathrm{d} y>0$, the dimensionless number $h_{0}$ may play a role opposite to that of $h_{1}$ : when the fluid viscosity $\tilde{\mu}_{\mathrm{f}}$ depends strongly on $\nu$ and the derivative $\mathrm{d} y / \mathrm{d} \tilde{z}$ is negative, then larger values of $h_{0}$ balance the effect of $h_{1}$, and tend to place the boundary layer at the top interface.

(iv) The constitutive quantities $\tilde{\mu}_{\mathrm{f}}$ and $\tilde{\beta}_{\mathrm{s}}$ and the dimensionless numbers $h_{0}$ and $h_{1}$ could be such that the $\mathfrak{h}$-functional maps the solid-volume fraction profile into a function vanishing at $\tilde{z}^{*}$ included in the interior of the interval or in a finite subset $V \subset[0,1]$. In this case, the boundary layer in which the high variations of $\nu$ are concentrated can occur either in the neighbourhood of $V$, at both the upper and lower interfaces or in the neighbourhood of $V$ as well as at the upper and lower interfaces.

These considerations underscore the prominent role played by the functional $\mathfrak{h}$ and the reason one can call it the boundary-layer localizing functional.

\section{(b) A criterion for the a priori determination of the localization of boundary layers}

In order to find a criterion for the a priori determination of the localization of the boundary layer, at least in those cases in which the boundary layers are located at the top or at the bottom interface, recall the boundary conditions (2.3) and (2.4) derived in detail in DH-II. In dimensionless form and subject to the steady-state conditions (2.5), they read

$$
\left.\begin{array}{c}
-h_{0} \tilde{\mu}_{\mathrm{f}}(y(0)) \frac{\mathrm{d} y}{\mathrm{~d} \tilde{z}}(0)+\frac{1}{y(0)} \tilde{\beta}_{\mathrm{s}}(y(0))-\frac{1}{y(0)-1} \frac{p_{\mathrm{i}}}{\left[\beta_{1}\right]}-\frac{y(0)}{y(0)-1} h_{3}=0, \quad \text { at } \tilde{z}=0, \\
-h_{0} \tilde{\mu}_{\mathrm{f}}(y(1)) \frac{\mathrm{d} y}{\mathrm{~d} \tilde{z}}(1)+\frac{1}{y(1)} \tilde{\beta}_{\mathrm{s}}(y(1))+\left(1-\alpha\left(\frac{y(1)-1}{y(1)}\right)^{l-1}\right) \frac{p_{\mathrm{i}}}{\left[\beta_{1}\right]}=0, \quad \text { at } \tilde{z}=1,
\end{array}\right\}
$$

where

$$
h_{3}:=\left(\frac{V_{\mathrm{f} 0} \hat{\rho}_{\mathrm{f}}}{\mathfrak{m}_{\mathrm{f}}^{\mathrm{b}}}\right)^{1 / 4} \frac{1}{\left[\beta_{1}\right]},
$$

and the drainage coefficient $\mathfrak{m}_{\mathrm{f}}^{\mathrm{b}}$ together with the interaction coefficients $\alpha$ and $l$ were introduced in DH-I and DH-II. Conditions (3.1) are consequences of the jump conditions of mass and linear momentum at the top and bottom interfaces of the draining layer, respectively. 
Recalling the first integral of (2.13) given by $(2.20)_{1}$, evaluating it at $y=y(1)$, replacing it in $(3.1)_{2}$, and subtracting the resulting expression from $(3.1)_{1}$, we obtain

$$
\begin{aligned}
-\int_{y(0)}^{y(1)} \mathfrak{d}\left(y_{1}\right) \mathrm{d} y_{1}+h_{0} h_{2}-(1 & \left.-\alpha\left(\frac{y(1)-1}{y(1)}\right)^{l-1}+\frac{1}{y(0)-1}\right) \frac{p_{\mathrm{i}}}{\left[\beta_{1}\right]} \\
& =\frac{1}{y(1)} \tilde{\beta}_{\mathrm{s}}(y(1))-\frac{1}{y(0)} \tilde{\beta}_{\mathrm{s}}(y(0))+\frac{y(0)}{y(0)-1} h_{3},
\end{aligned}
$$

which is one relation between the values $y(1)$ and $y(0)$. Now, summing up the two relations in (3.1), we obtain

$$
\begin{aligned}
h_{0} \tilde{\mu}_{\mathrm{f}}(y(0)) \frac{\mathrm{d} y}{\mathrm{~d} \tilde{z}}(0)+ & h_{0} \tilde{\mu}_{\mathrm{f}}(y(1)) \frac{\mathrm{d} y}{\mathrm{~d} \tilde{z}}(1) \\
=\frac{1}{y(0)} \tilde{\beta}_{\mathrm{s}}(y(0)) & +\frac{1}{y(1)} \tilde{\beta}_{\mathrm{s}}(y(1))-\frac{y(0)}{y(0)-1} h_{3} \\
& +\left(1-\alpha\left(\frac{y(1)-1}{y(1)}\right)^{l-1}-\frac{1}{y(0)-1}\right) \frac{p_{\mathrm{i}}}{\left[\beta_{1}\right]} .
\end{aligned}
$$

The terms on its left-hand side also arise in the form (2.21) of the $\mathfrak{h}$-functional and, consequently, may be replaced there by the right-hand side of (3.3). This process yields the expression

$$
\begin{aligned}
& \mathfrak{h}(\cdot, \tilde{z}):=\mathfrak{d}(\nu(y))+\frac{1}{2} \frac{\mathrm{d} \ln \tilde{\mu}_{\mathrm{f}}}{\mathrm{d} y}\left(-\int_{y(1)}^{y} \mathfrak{d}\left(y_{1}\right) \mathrm{d} y_{1}-\int_{y(0)}^{y} \mathfrak{d}\left(y_{1}\right) \mathrm{d} y_{1}\right. \\
& -h_{0} h_{2}(2 \tilde{z}-1)+\frac{1}{y(0)} \tilde{\beta}_{\mathrm{s}}(y(0))+\frac{1}{y(1)} \tilde{\beta}_{\mathrm{s}}(y(1)) \\
& \left.+\left(1-\alpha\left(\frac{y(1)-1}{y(1)}\right)^{l-1}-\frac{1}{y(0)-1}\right) \frac{p_{\mathrm{i}}}{\left[\beta_{1}\right]}-\frac{y(0)}{y(0)-1} h_{3}\right) .
\end{aligned}
$$

Therefore, once the constitutive equations for $\tilde{\beta}_{\mathrm{s}}$ and $\tilde{\mu}_{\mathrm{f}}$ are assigned, the sign of the image of the $\mathfrak{h}$-functional, when evaluated on the profile $y(\tilde{z})$ assuming the boundary values $y(0)$ and $y(1)$, is, in principle, determinable once a further relation between $y(0)$ and $y(1)$ is added to (3.2).

To find such a relation, recall that $(2.24)_{2}$ provides an accurate estimate for the derivative $\mathrm{d} y / \mathrm{d} \tilde{z}$ at the interface at which the boundary layer does not arise. This value can be used in that relation (3.1) where the boundary does not occur. Therefore, we have the following alternatives.

(i) If the boundary layer is tentatively assumed to be localized at $\tilde{z}=1$, then the second equation to be added to (3.2) will be

$$
\frac{1}{y(0)} \tilde{\beta}_{\mathrm{s}}(y(0))-\frac{1}{y(0)-1} \frac{p_{\mathrm{i}}}{\left[\beta_{1}\right]}-\frac{y(0)}{y(0)-1} h_{3}=\left(\frac{h_{0}^{2} h_{2}}{\mathfrak{h}(\cdot, 0)}\right) \tilde{\mu}_{\mathrm{f}}(y(0)) .
$$

Note that $\mathfrak{h}(\cdot, 0)$ can be computed from $(3.4)$ by setting $\tilde{z}=0$. Thus, by solving the nonlinear system $(3.2),(3.5)$, one finds values for $y(0)$ and $y(1)$, with the aid of which the $\mathfrak{h}$-functional can be evaluated for all $\tilde{z} \in[0,1]$. If it is negative, then the boundary layer will indeed be localized at $\tilde{z}=1$. 
(ii) If the boundary layer is tentatively assumed to be localized at $\tilde{z}=0$, then the second equation to be added to (3.2) will be

$$
\frac{1}{y(1)} \tilde{\beta}_{\mathrm{s}}(y(1))+\left(1-\alpha\left(\frac{y(1)-1}{y(1)}\right)^{l-1}\right) \frac{p_{\mathrm{i}}}{\left[\beta_{1}\right]}=\left(\frac{h_{0}^{2} h_{2}}{\mathfrak{h}(\cdot, 1)}\right) \tilde{\mu}_{\mathrm{f}}(y(1)) .
$$

Thus, by solving the system (3.2), (3.6), one finds different values for $y(0)$ and $y(1)$. With these, the $\mathfrak{h}$-functional must be positive for all $\tilde{z} \in[0,1]$ in order that the boundary layer is indeed localized at $\tilde{z}=0$.

Appendix A shows how the volume fraction is constructed within the boundary layer once its location has been determined.

A more complex analysis, which we postpone to further investigations, is necessary if the $\mathfrak{h}$-functional does not show a definite sign in the interval $[0,1]$.

\section{Viscosity in isolation}

Consider the central differential equation (2.13) when the free energy is not a function of the solid-volume fraction, i.e. for vanishing thermodynamic pressure. In that case, (2.13) becomes

$$
\left.\begin{array}{c}
\frac{\mathrm{d}}{\mathrm{d} \tilde{z}}\left(\tilde{\mu}_{\mathrm{f}}(\nu) \frac{\mathrm{d} y}{\mathrm{~d} \tilde{z}}\right)+\frac{1}{\varepsilon_{\mathrm{c}}} \frac{\mathrm{d} y}{\mathrm{~d} \tilde{z}}=\frac{1}{\varepsilon}, \\
\varepsilon:=\frac{1}{h_{2}} \in \frac{1}{3.75}\left[10^{-15}, 10^{-13}\right], \\
\frac{h_{0}}{h_{1}}=\frac{4\left[\mu_{\mathrm{f}}\right]}{3 \hat{\rho}_{\mathrm{f}}\left|V_{\mathrm{f} 0}\right|[H]} \in\left[2 \times 10^{-3}, 10^{-1}\right],
\end{array}\right\}
$$

in which $\varepsilon$ and $\varepsilon_{\mathrm{c}}$ are both small. If we rescale the equation by the stretching transformation,

$$
\zeta=\frac{1}{\sqrt{ } \varepsilon} \tilde{z}, \quad \frac{\mathrm{d}}{\mathrm{d} \tilde{z}}=\frac{1}{\sqrt{ } \varepsilon} \frac{\mathrm{d}}{\mathrm{d} \zeta},
$$

(4.1) transforms into

$$
\frac{\mathrm{d}}{\mathrm{d} \zeta}\left(\tilde{\mu}_{\mathrm{f}}(\nu) \frac{\mathrm{d} y}{\mathrm{~d} \zeta}\right)+\eta \frac{\mathrm{d} y}{\mathrm{~d} \zeta}=1, \quad \eta:=\frac{\varepsilon^{1 / 2}}{\varepsilon_{\mathrm{c}}},
$$

with typical values of $\eta$ being very small, $\eta \in\left[10^{-18}, 10^{-16}\right]$. Equation (4.3) shows that incorporating vertical convection boils down to solving a regular perturbation problem. In addition, (4.3) also points at an important conclusion that applies when the thermodynamic pressure is ignored: there is no true boundary layer; indeed, with the stretching transformation (4.2), an ODE emerges that (for $\eta=0$, i.e. when vertical convection is ignored) does not give rise to the use of matched asymptotics. In other words, if one wants to maintain the notion of boundary layer, this boundary layer fills the entire region. This will be reflected in the solution (4.9) corresponding to (4.3) when $\eta=0$.

A first integral of (4.3) is

$$
\tilde{\mu}_{\mathrm{f}}(\nu) \frac{\mathrm{d} y}{\mathrm{~d} \zeta}+\eta y=\zeta+\left(C_{1}+\eta C_{2}+\cdots\right),
$$


in which $\left(C_{1}+\eta C_{2}+\cdots\right)$ is a constant of integration that has been split into an $O(1)$ and $O(\eta)$ term for convenience. Seeking a perturbation solution in the form

$$
y=\stackrel{0}{y}+\eta \stackrel{1}{y}+\cdots,
$$

substituting this expansion into (4.4) and collecting terms of equal order in $\eta$, we arrive at the equations

$$
\left.\begin{array}{c}
\tilde{\mu}_{\mathrm{f}}(\stackrel{0}{y}) \frac{\mathrm{d} \stackrel{0}{y}}{\mathrm{~d} \tilde{z}}=\frac{\tilde{z}}{\varepsilon}+\frac{C_{1}}{\sqrt{ } \varepsilon}, \\
\frac{\mathrm{d}}{\mathrm{d} \tilde{z}}\left(\tilde{\mu}_{\mathrm{f}}(\stackrel{0}{y}) \stackrel{1}{y}\right)=-\frac{\stackrel{0}{y}+C_{2}}{\sqrt{ } \varepsilon}, \\
\vdots
\end{array}\right\}
$$

which must be subjected to the boundary conditions

$$
\left.\begin{array}{lll}
\zeta=0, & \stackrel{0}{y}=y_{0}, & \stackrel{1}{y}=0, \\
\zeta=1 / \sqrt{ } \varepsilon, & \stackrel{0}{y}=y_{h}, & \stackrel{1}{y}=0 .
\end{array}\right\}
$$

Solving the zeroth-order equation is physically equivalent to ignoring the vertical fluid convection and yields the solution

$$
M\left(\stackrel{0}{y}, y_{h}\right)=\frac{1}{2} \zeta^{2}+C_{1} \zeta+C_{3}, \quad M\left(y, y_{h}\right)=\int_{y_{h}}^{y} \tilde{\mu}_{\mathrm{f}}(\xi) \mathrm{d} \xi
$$

or, upon the imposition of the boundary conditions and transformation back to the $\tilde{z}$ variable,

$$
M\left(\stackrel{0}{y}, y_{h}\right)=(1 / 2 \varepsilon)\left(\tilde{z}^{2}-\tilde{z}\right)+M\left(y_{0}, y_{h}\right)(1-\tilde{z})
$$

Let

$$
K(\tilde{z}):=M\left(\stackrel{0}{y}(\tilde{z}), y_{h}\right) .
$$

Then (4.9) may take the alternative form

$$
K(\tilde{z})=(\tilde{z}-1)\left((\tilde{z} / 2 \varepsilon)-M\left(y_{0}, y_{h}\right)\right) .
$$

The zeros of this function lie at $\tilde{z}_{1}=1$ and $\tilde{z}_{2}=2 \varepsilon M\left(y_{0}, y_{h}\right)>0$, and its minimum at $\tilde{z}_{\min }=\frac{1}{2}+\varepsilon M\left(y_{0}, y_{h}\right)$ is given by

$$
K_{\min }=K\left(\tilde{z}_{\min }\right)=-(1 / 2 \varepsilon)\left(\frac{1}{2}-\varepsilon M\left(y_{0}, y_{h}\right)\right)^{2}<0 .
$$

Two cases may, in principle, arise:

(i) the smaller of the two roots is at $\tilde{z}=\tilde{z}_{2}$, the larger at $\tilde{z}=\tilde{z}_{1}=1$, and, thus, the negative $K_{\min }$ is assumed within the interval $\left[\tilde{z}_{2}, \tilde{z}_{1}\right]$, implying that $\tilde{\mu}_{\mathrm{f}}(y(\nu))$ must be negative for some $\nu \in[0,1]$, which is obviously unphysical;

(ii) $\tilde{z}_{2}>\tilde{z}_{1}$, or

$$
1<2 \varepsilon M\left(y_{0}, y_{h}\right)
$$

in which case $\tilde{\mu}_{\mathrm{f}}(y(\nu))>0, \forall \nu \in[0,1]$, as is physically expected. 
Consider as an example constant fluid viscosity $\tilde{\mu}_{\mathrm{f}}=1$. Then

$$
2 \varepsilon M\left(y_{0}, y_{h}\right)=2 \varepsilon\left(y_{0}-y_{h}\right)>0 .
$$

This quantity is, for all physically admissible values of $y_{0}$ and $y_{h}$, certainly smaller than unity, implying that selecting a constant viscosity cannot possibly be a physically acceptable choice (see also DH-III).

Thus, $\tilde{\mu}_{\mathrm{f}}(y)$ must be a 'true' function of its argument and such that (4.12) can be fulfilled. With this in mind, it is reasonable to suppose that $\tilde{\mu}_{\mathrm{f}}$ becomes large as $\nu$ approaches an upper bound, say $\nu_{\mathrm{L}}<1$, but $\tilde{\mu}_{\mathrm{f}} \gtrsim 1$ whenever $\nu$ is distant from this bound, $\nu \ll \nu_{\mathrm{L}}$, where L stands for 'limit'. Such a behaviour is qualitatively described by

$$
\tilde{\mu}_{\mathrm{f}}=1-\frac{\mathfrak{a}}{\varepsilon} \frac{1}{y-y_{\mathrm{L}}}
$$

where $\mathfrak{a}$ and $y_{\mathrm{L}}=y\left(\nu_{\mathrm{L}}\right)$ are constants. With this choice, one may easily demonstrate that (4.12) implies

$$
2 \varepsilon M\left(y_{0}, y_{h}\right)=2 \mathfrak{a} \ln \left(\frac{y_{\mathrm{L}}-y_{h}}{y_{\mathrm{L}}-y_{0}}\right)+2 \varepsilon\left(y_{0}-y_{\mathrm{L}}\right)>1,
$$

or, approximately,

$$
\frac{y_{\mathrm{L}}-y_{h}}{y_{\mathrm{L}}-y_{0}}>\exp \left(\frac{1}{2 \mathfrak{a}}\right) .
$$

This formula may be used to find estimates for $y_{\mathrm{L}}$ and $\mathfrak{a}$.

The examples show that for the model to be physically realistic, the fluid viscosity must be strongly dependent upon the solid-volume fraction; for most solid concentrations below a certain upper bound it is nearly constant, but as this upper limit $\nu_{\mathrm{L}}<1$ is reached, it becomes large, infinitely large as $\nu \rightarrow \nu_{\mathrm{L}}$, according to (4.13).

Consider next the first-order equation $(4.6)_{2}$; its integral is given by

$$
\stackrel{1}{y}=-\frac{1}{\tilde{\mu}_{\mathrm{f}}(\stackrel{0}{y})}\left\{\int_{0}^{\zeta} \stackrel{0}{y}(\xi) \mathrm{d} \xi-C_{2} \zeta\right\}+C_{4},
$$

and this becomes, when the boundary conditions (4.7) are imposed,

$$
\stackrel{1}{y}=-\frac{1}{\tilde{\mu}_{\mathrm{f}}(y)}\left\{\int_{0}^{\tilde{z} / \sqrt{ } \varepsilon}{ }_{0}^{y}(\xi) \mathrm{d} \xi-\tilde{z} \int_{0}^{1 / \sqrt{ } \varepsilon} \stackrel{0}{y}(\xi) \mathrm{d} \xi\right\} .
$$

\section{Viscosity functions achieving the 'boundary-layer flip'}

There still remains to be answered the question of whether there exists, in principle, a viscosity function $\tilde{\mu}_{\mathrm{f}}(y)$ for which the $\mathfrak{h}$-functional can change sign because convection is taken into account. This is possible provided that

$$
h_{0} \frac{\mathrm{d} \tilde{\mu}_{\mathrm{f}}}{\mathrm{d} y} \frac{\mathrm{d} y}{\mathrm{~d} \tilde{z}}+h_{1}>0
$$


or, with the estimate (A 10) of Appendix A, where the maximum of $|\mathrm{d} y / \mathrm{d} \tilde{z}|$ is computed:

$$
1>\left(y_{0}-y_{h}\right) \frac{\mathrm{d}}{\mathrm{d} y}\left(\ln \tilde{\mu}_{\mathrm{f}}\right) .
$$

Substituting the ansatz (4.13), it is straightforward to show that

$$
\frac{(1 / 2 \varepsilon)-\left(y_{0}-y_{h}\right)}{\ln \left[\left(y_{\mathrm{L}}-y_{h}\right) /\left(y_{\mathrm{L}}-y_{0}\right)\right]}<\frac{\mathfrak{a}}{\varepsilon}<\frac{\left(y_{\mathrm{L}}-y_{0}\right)^{2}}{\left[\left(y_{0}-y_{h}\right)-\left(y_{\mathrm{L}}-y_{0}\right)\right]}
$$

Here we have also added the left inequality, which is a direct consequence of (4.14). Physically, $y_{\mathrm{L}}>y_{0}>y_{h}$, and $y_{\mathrm{L}}-y_{0}=: \delta$ is small. If this quantity is introduced in (5.3), it takes the form

$$
\underbrace{\frac{(1 / 2 \varepsilon)-\left(y_{0}-y_{h}\right)}{\ln \left[\left(y_{0}-y_{h}+\delta\right) / \delta\right]}}_{\text {positive, very large }}<\frac{\mathfrak{a}}{\varepsilon}<\underbrace{\frac{\delta^{2}}{\left[\left(y_{0}-y_{h}\right)-\delta\right]}}_{\text {positive, very small }} .
$$

Consequently, both inequalities cannot be satisfied simultaneously; vertical convection cannot, in this case, change the structure of the solution.

If, on the other hand, we select

$$
\tilde{\mu}_{\mathrm{f}}=1+\frac{\mathfrak{a}}{\varepsilon}\left(\frac{1}{y_{\mathrm{L}}-y}\right)^{m}, \quad m \in(0,1),
$$

and repeat the above analysis, the following chain of inequalities is obtained,

$$
\frac{(1-m)\left((1 / 2 \varepsilon)-\left(y_{0}-y_{h}\right)\right)}{\left[\left(y_{0}-y_{h}+\delta\right)^{1-m}-\delta^{1-m}\right]}<\frac{\mathfrak{a}}{\varepsilon}<\frac{\delta^{m+1}}{\left[m\left(y_{0}-y_{h}\right)-\delta\right]},
$$

or, a fortiori,

$$
\frac{(1-m)}{2 \varepsilon\left(y_{0}-y_{h}\right)^{1-m}}<\frac{\mathfrak{a}}{\varepsilon}<\frac{\delta^{m+1}}{m\left(y_{0}-y_{h}\right)} .
$$

If we choose $m=\varepsilon \delta^{m+1}\left(y_{0}-y_{h}\right)^{-m}>0$, which is very small, then (5.7) is verified by

$$
(1-m)<\frac{\mathfrak{a}}{\varepsilon}<1 .
$$

We have thus shown that there exists an exponent $m>0$ (although still very small), such that the constitutive function for $\tilde{\mu}_{\mathrm{f}}$ (see (5.5)) obeys both inequalities (5.6). A change of the boundary layer in the solid-volume fraction profile from the top to the bottom interface is, therefore, possible in our model, and uses a fluid viscosity function (5.5), with $\mathfrak{a} / \varepsilon$ verifying (5.8), and $m>0$ and very small. This would mean that $\tilde{\mu}_{\mathrm{f}}$ is effectively constant for all $y<y_{\mathrm{L}}$ and suddenly becomes infinitely large as $y \rightarrow y_{\mathrm{L}}$. This Dirac-type behaviour is obviously very singular but physically unavoidable if the boundary-layer flip is to occur. 


\section{Horizontal velocity fields}

In this section, by using approximate integration techniques, we show that the horizontal velocities of the solid and fluid constituents depend on the mean of the solid and fluid viscosities outside the boundary layer, and, thus, also depend on the location of the boundary layer itself. To demonstrate this, the differential equations $(2.1)_{5,6}$ for $u_{\mathrm{s}}$ and $u_{\mathrm{f}}$,

$$
\left.\begin{array}{l}
\left(\mu_{\mathrm{s}} u_{\mathrm{s}}^{\prime}\right)^{\prime}-\nu(1-\nu) \tilde{\alpha}\left(u_{\mathrm{s}}-u_{\mathrm{f}}\right)=\nu \frac{\partial p}{\partial x}+\hat{\rho}_{\mathrm{s}} \nu w_{\mathrm{s}} u_{\mathrm{s}}^{\prime}, \\
\left(\mu_{\mathrm{f}} u_{\mathrm{f}}^{\prime}\right)^{\prime}+\nu(1-\nu) \tilde{\alpha}\left(u_{\mathrm{s}}-u_{\mathrm{f}}\right)=(1-\nu) \frac{\partial p}{\partial x}+\hat{\rho}_{\mathrm{f}}(1-\nu) w_{\mathrm{f}} u_{\mathrm{f}}^{\prime},
\end{array}\right\}
$$

will be approximately integrated for (i) $\partial p / \partial x=0$, i.e. vanishing horizontal gradient of the (incumbent) pressure; (ii) $w_{\mathrm{s}}=0$ (no abrasion); and (iii) under the assumption that the vertical convective terms of the fluid constituent can be ignored. Furthermore, for simplicity it will be assumed that $\mu_{\mathrm{s}}$ can be assumed to be constant, at least outside the boundary layer, and that equation (6.1) is only integrated over that part of the mixture layer that does not contain the boundary layer. $\dagger$ On this basis, the fluid viscosity outside the boundary layer can also be assumed to be constant, as is the volume fraction.

With these restrictive conditions, equation (6.1) implies

$$
\left.\begin{array}{l}
\left(\mu_{\mathrm{s}} u_{\mathrm{s}}^{\prime}\right)^{\prime}-\nu(1-\nu) \tilde{\alpha}\left(u_{\mathrm{s}}-u_{\mathrm{f}}\right)=0 \\
\left(\mu_{\mathrm{f}} u_{\mathrm{f}}^{\prime}\right)^{\prime}+\nu(1-\nu) \tilde{\alpha}\left(u_{\mathrm{s}}-u_{\mathrm{f}}\right)=0
\end{array}\right\}
$$

from which, with appropriate combinations, the following ODEs may be deduced

$$
\left.\begin{array}{r}
\left(\mu_{\mathrm{s}} u_{\mathrm{s}}^{\prime}+\mu_{\mathrm{f}} u_{\mathrm{f}}^{\prime}\right)^{\prime}=0, \\
\left(u_{\mathrm{s}}-u_{\mathrm{f}}\right)^{\prime \prime}-\underbrace{\frac{\mu_{\mathrm{s}}+\mu_{\mathrm{f}}}{\mu_{\mathrm{f}} \mu_{\mathrm{s}}} \nu(1-\nu) \tilde{\alpha}}_{=: c^{2}}\left(u_{\mathrm{s}}-u_{\mathrm{f}}\right)=0 .
\end{array}\right\}
$$

Because outside the boundary layer $\nu$ is effectively constant, these differential equations are linear with constant coefficients. Their integrals read

$$
\left.\begin{array}{c}
\mu_{\mathrm{s}} u_{\mathrm{s}}+\mu_{\mathrm{f}} u_{\mathrm{f}}=A z+B \\
u_{\mathrm{s}}-u_{\mathrm{f}}=C \exp (c z)+D \exp (-c z)
\end{array}\right\}
$$

where $c$ is identified in (6.3).

It will now be assumed that the boundary layer is so thin that it can be ignored when the boundary conditions are imposed. At the bottom interface, the no-slip conditions will be applied,

$$
u_{\mathrm{s}}(0)=u_{\mathrm{f}}(0)=0,
$$

and at the top interface the applied shear traction $S$ will be distributed among the constituents according to

$$
\mu_{\mathrm{s}} u_{\mathrm{s}}^{\prime}=2 \alpha \nu^{l} S, \quad \mu_{\mathrm{f}} u_{\mathrm{f}}^{\prime}=2\left(1-\alpha \nu^{l}\right) S, \quad \text { at } z=H .
$$

$\dagger$ We suppose here Newtonian rheology with viscosity that may depend on volume fraction, even though experiments suggest a power law rheology (see Vulliet \& Hutter 1988).

Proc. R. Soc. Lond. A (1999) 
For a justification, the reader is referred to DH-I and DH-II. With the conditions (6.5) and (6.6), it is then straightforward to show that

$$
B=0, \quad A=2 S / H, \quad C=-D, \quad D=\frac{2}{c} \frac{\left(\alpha \nu^{l} / \mu_{\mathrm{s}}\right)-\left[\left(1-\alpha \nu^{l}\right) / \mu_{\mathrm{f}}\right]}{\exp (-c H)+\exp (c H)} S .
$$

With these, $u_{\mathrm{s}}$ and $u_{\mathrm{f}}$ may be isolated:

$$
\begin{aligned}
& u_{\mathrm{s}}(z)=\left\{\frac{2}{\mu_{\mathrm{s}}+\mu_{\mathrm{f}}} z+\frac{2 \mu_{\mathrm{f}}}{c\left(\mu_{\mathrm{s}}+\mu_{\mathrm{f}}\right)}\left(\alpha \nu^{l}-\left(1-\alpha \nu^{l}\right)\right) \frac{\exp (-c z)-\exp (c z)}{\exp (-c H)+\exp (c H)}\right\} S, \\
& u_{\mathrm{f}}(z)=\left\{\frac{2}{\mu_{\mathrm{s}}+\mu_{\mathrm{f}}} z-\frac{2 \mu_{\mathrm{s}}}{c\left(\mu_{\mathrm{s}}+\mu_{\mathrm{f}}\right)}\left(\alpha \nu^{l}-\left(1-\alpha \nu^{l}\right)\right) \frac{\exp (-c z)-\exp (c z)}{\exp (-c H)+\exp (c H)}\right\} S .
\end{aligned}
$$

The first terms on the right-hand side are the classical linear shear profiles of Newtonian fluids, and it is interesting that they are the same for the solid and the fluid. This reflects the barycentric motion for which the interaction force is not active. The remaining terms are exactly the manifestation of the latter; however, because $c$ is very large outside the boundary layer, the second term influences the velocity profiles at most very close to the top boundary.

\section{Consequences of the compactness of the till layer}

The model on which the results of the previous sections are based was motivated by DH-I, DH-II and DH-III. There, suitable constitutive equations and the numerical values for all introduced constitutive constants were chosen as listed in table 1 . The only exception was the constitutive equation for the fluid viscosity, a formula for which was proposed above. With $\tilde{\mu}_{\mathrm{f}}$ given by (5.5), we may deduce

$$
\frac{\mathrm{d}}{\mathrm{d} y}\left\{\ln \left(1+\frac{\mathfrak{a}}{\varepsilon}\left(\frac{1}{y_{\mathrm{L}}-y}\right)^{m}\right)\right\}=\frac{\mathfrak{a}}{\varepsilon} m \frac{1}{\left(y_{\mathrm{L}}-y\right)\left[\left(y_{\mathrm{L}}-y\right)^{m}+(\mathfrak{a} / \varepsilon)\right]} .
$$

Since, according to (5.6) and (5.7), a $/ \varepsilon \rightarrow 1^{-}$and $m \rightarrow 0^{+}$, this may be approximated as $m /\left(y_{\mathrm{L}}-y\right)$; so, as long as $y$ does not become too close to $y_{\mathrm{L}}$, the difference

$$
1-\left(y_{0}-y_{h}\right) \frac{\mathrm{d}}{\mathrm{d} y} \ln \tilde{\mu}_{\mathrm{f}}=1^{-},
$$

is not much smaller than unity. Therefore,

$$
h_{0} \frac{\mathrm{d} \tilde{\mu}_{\mathrm{f}}}{\mathrm{d} y} \frac{\mathrm{d} y}{\mathrm{~d} \tilde{z}}+h_{1} \simeq\left(1^{-}\right) h_{1}
$$

and

$$
\mathfrak{h}(\cdot, \tilde{z})=\left(1^{-}\right) h_{1}+h(\nu)
$$

can change sign from being negative to positive by making $h_{1}$ sufficiently large. As the second term on the right-hand side of (7.4) is of the order of $10^{-14}, h_{1}$ must be of that order of magnitude to achieve such a change of sign. With the definition $(2.15)_{2}$ and the numerical values of table 1 , this yields a water flow rate per unit area $\left|V_{\mathrm{f} 0}\right|$ of at most a few tens of centimetres per year. This is a very 'reasonable' number, implying that for realistic melting rates of ice close to the bottom of ice sheets, the 
water production to be drawn to the sediment can, via its convective motion, cause the boundary layer to flip from the top interface to the bottom interface. When this happens, the compactness of the layer will be much looser than otherwise, giving rise in turn to larger effective fluidity against horizontal shear, so that associated slip velocities may become large when this boundary-layer flip occurs.

This behaviour can simply be inferred from the representation (5.5) of the fluid viscosity. If the boundary layer is at the top, $y(\nu)$ is close to $y_{\mathrm{L}}$ through most of the sediment layer, and $\tilde{\mu}_{\mathrm{f}}$ is nearly constant except in the small boundary layer. With the horizontal velocity of the sediment given by approximately

$$
u_{\mathrm{s}}(z) \simeq\left[2 S /\left(\mu_{\mathrm{s}}+\mu_{\mathrm{f}}\right)\right] z .
$$

This implies that

$$
u_{\mathrm{s}}^{\mathrm{BLT}}(H):=\left[2 S /\left(\mu_{\mathrm{s}}+\mu_{\mathrm{f}}\right)^{\max }\right] H
$$

is relatively small, since $\left(\mu_{\mathrm{s}}+\mu_{\mathrm{f}}\right)^{\max }$ (i.e. the viscosities estimated for the values of $\nu$ assumed when the boundary layer is at the top) is large as $\nu \rightarrow \nu_{\mathrm{L}}$. If, alternatively, the boundary layer is at the bottom, then $\nu$ is distant from $\nu_{\mathrm{L}}$ throughout most of the sediment layer, the sediment velocity

$$
u_{\mathrm{s}}^{\mathrm{BLB}}(H):=\left[2 S /\left(\mu_{\mathrm{s}}+\mu_{\mathrm{f}}\right)^{\mathrm{min}}\right] H
$$

is large, since $\left(\mu_{\mathrm{s}}+\mu_{\mathrm{f}}\right)^{\mathrm{min}}$ (i.e. the viscosities estimated for the values of $\nu$ assumed when the boundary layer is at the bottom). In fact, we may grossly estimate

$$
\frac{u_{\mathrm{s}}^{\mathrm{BLB}}(H)}{u_{\mathrm{s}}^{\mathrm{BLT}}(H)} \simeq \frac{\left(\mu_{\mathrm{s}}+\mu_{\mathrm{f}}\right)^{\max }}{\left(\mu_{\mathrm{s}}+\mu_{\mathrm{f}}\right)^{\min }}
$$

which is large if $\nu_{\mathrm{L}}-\nu_{0}$ is small.

In brief we summarize: provided the fluid viscosity is chosen according to (5.5) and (5.8) we have

1. the average solid-volume fraction of the till layer is lower when the boundary layer is located at the bottom;

2. for ice-melting rates of a few tens of centimetres per year, the boundary layer is located at the bottom of the till layer; and

3. the shear flow in the till layer, in the presence of a given shear action, is greater when the boundary layer is at the bottom.

These results demonstrate that the model on which the present paper is based predicts a possible destabilizing mechanism of a pressurized and sheared drained layer of a soil water mixture. Numerical results that underline these claims quantitatively will be presented elsewhere.

We thank Richard Hindmarsh and an anonymous referee for their critical comments of an earlier manuscript.

Proc. R. Soc. Lond. A (1999) 


\section{Appendix A.}

Here we construct the boundary-layer solutions. Consider equation (2.13) and assume the $\mathfrak{h}$-functional to be positive so that the boundary layer is at $\tilde{z}=0$. Introduce the boundary-layer coordinate

$$
\zeta=\frac{1}{h_{0}} \tilde{z}, \quad \frac{\mathrm{d}}{\mathrm{d} \tilde{z}}=\frac{1}{h_{0}} \frac{\mathrm{d}}{\mathrm{d} \zeta} .
$$

Then equation (2.13) takes the form

$$
\frac{\mathrm{d}}{\mathrm{d} \zeta}\left(\tilde{\mu}_{\mathrm{f}}(y) \frac{\mathrm{d} y}{\mathrm{~d} \zeta}\right)+\mathfrak{d}(\nu(y)) \frac{\mathrm{d} y}{\mathrm{~d} \zeta}=h_{0}^{2} h_{2}
$$

which can also be written as

$$
\frac{\mathrm{d}}{\mathrm{d} \zeta}\left(\tilde{\mu}_{\mathrm{f}}(y) \frac{\mathrm{d} y}{\mathrm{~d} \zeta}+\mathfrak{D}\left(y ; y^{*}\right)\right)=h_{0}^{2} h_{2},
$$

where

$$
\mathfrak{D}\left(y ; y^{*}\right):=\int_{y^{*}}^{y} \mathfrak{d}(\nu(\bar{y})) \mathrm{d} \bar{y} .
$$

The solution of (A 3$)$ as $h_{0}^{2} h_{2} \rightarrow 0$, is given by

$$
\tilde{\mu}_{\mathrm{f}}(y) \frac{\mathrm{d} y}{\mathrm{~d} \zeta}=C_{1}-\mathfrak{D}\left(y ; y^{*}\right),
$$

and a further integration yields

$$
\zeta=\int_{1}^{y} \frac{\tilde{\mu}_{\mathrm{f}}(\bar{y})}{C_{1}-\mathfrak{D}\left(\bar{y} ; y^{*}\right)} \mathrm{d} \bar{y}+C_{2} .
$$

At $\zeta=0$, one must have $y=y_{0}$, which determines $C_{2}$; as a result, (A 6) becomes

$$
\zeta=\int_{y_{0}}^{y} \frac{\tilde{\mu}_{\mathrm{f}}(\bar{y})}{C_{1}-\mathfrak{D}\left(\bar{y} ; y^{*}\right)} \mathrm{d} \bar{y} .
$$

Matching this for $\zeta=\infty$ with the outer solution $y=y_{h}$ yields

$$
\infty=\int_{y_{0}}^{y_{h}} \frac{\tilde{\mu}_{\mathrm{f}}(\bar{y})}{C_{1}-\mathfrak{D}\left(\bar{y} ; y^{*}\right)} \mathrm{d} \bar{y} .
$$

Provided $\tilde{\mu}_{\mathrm{f}}>0$ is a monotone function of its argument, (A 8) implies $C_{1}=\mathfrak{D}\left(y_{h} ; y^{*}\right)$, so that the inner solution expressed in the outer variable takes the form

$$
\tilde{z}=h_{0} \int_{y_{0}}^{y} \frac{\tilde{\mu}_{\mathrm{f}}(\bar{y})}{\mathfrak{D}\left(y_{h} ; y^{*}\right)-\mathfrak{D}\left(\bar{y} ; y^{*}\right)} \mathrm{d} \bar{y}=-h_{0} \int_{y_{0}}^{y}\left[\tilde{\mu}_{\mathrm{f}}(\bar{y}) /\left(\int_{y_{h}}^{\bar{y}} \mathfrak{d}(x) d x\right)\right] \mathrm{d} \bar{y} .
$$

This solution also allows the evaluation of the derivative $\mathrm{d} y / \mathrm{d} \tilde{z}$; indeed, from (A 5) with $C_{1}$ given as above, one obtains

$$
\frac{\mathrm{d} y}{\mathrm{~d} \tilde{z}}=\frac{1}{h_{0} \tilde{\mu}_{\mathrm{f}}(y)}\left(\mathfrak{D}\left(y_{h} ; y^{*}\right)-\mathfrak{D}\left(y ; y^{*}\right)\right)=-\frac{1}{h_{0} \tilde{\mu}_{\mathrm{f}}(y)} \int_{y_{h}}^{y} \mathfrak{d}(x) \mathrm{d} x .
$$


If $\mathfrak{h}$ is negative, then the boundary layer is at $\tilde{z}=1$. If we then let the boundarylayer coordinate be

$$
\zeta=\frac{1}{h_{0}}(1-\tilde{z}), \quad \frac{\mathrm{d}}{\mathrm{d} \tilde{z}}=-\frac{1}{h_{0}} \frac{\mathrm{d}}{\mathrm{d} \zeta},
$$

and define

$$
\mathfrak{D}\left(y ; y^{*}\right):=-\int_{y^{*}}^{y} \mathfrak{d}(\nu(\bar{y})) \mathrm{d} \bar{y},
$$

the boundary-layer equation again takes the form (A 3), and the inner solution in terms of the outer variable now reads

$$
1-\tilde{z}=-h_{0} \int_{y_{h}}^{y}\left[\tilde{\mu}_{\mathrm{f}}(\bar{y}) /\left(\int_{y_{0}}^{\bar{y}} \mathfrak{d}(x) \mathrm{d} x\right)\right] \mathrm{d} \bar{y},
$$

and

$$
\frac{\mathrm{d} y}{\mathrm{~d} \tilde{z}}=\frac{1}{h_{0} \tilde{\mu}_{\mathrm{f}}(y)} \int_{y_{0}}^{y} \mathfrak{d}(x) \mathrm{d} x
$$

\section{References}

Alley, R. B., Blankenship, D. D., Bentley, C. R. \& Rooney, S. T. $1987 a$ Till beneath ice stream B, 3. Till deformation: evidence and implications. J. Geophys. Res. 92, 8921-8929.

Alley, R. B., Blankenship, D. D., Bentley, C. R. \& Rooney, S. T. $1987 b$ Till beneath ice stream B, 4. A coupled ice-till flow model. J. Geophys. Res. 92, 8931-8940.

Boulton, G. S. \& Hindmarsh, R. C. A. 1987 Sediment deformation beneath glaciers: rheology and geological consequences. J. Geophys. Res. 92, 9059-9082.

Bush, A. W. 1992 Perturbation methods for engineers and scientists. Boca Raton, FL: CRC.

Clarke, G. K. C. 1987 Subglacial till: a physical framework for its properties and processes. J. Geophys. Res. 92, 9023-9036.

de Boer, R. \& Ehlers, W. 1990 The development of the concept of effective stresses. Acta Mechanica 83, 77-92.

dell'Isola, F. \& Hutter, K. $1998 a$ What are the dominant thermomechanical processes in the basal sediment layer of large ice sheets? Proc. R. Soc. Lond. A 454, 1169-1195.

dell'Isola, F. \& Hutter, K. $1998 b$ A qualitative analysis of the dynamics of a sheared and pressurized layer of saturated soil. Proc. R. Soc. Lond. A 454, 3105-3120.

dell'Isola, F. \& Hutter, K. 1999 A free moving boundary problem for the till below large ice sheets. Free boundary problems, theory and applications. In Proc. Conf. in Heraclion. (In the press.)

Hutter, K., Jöhnk, K. \& Svendsen, B. 1994 On interfacial transition conditions in two phase gravity flow. Z. Angew. Math. Phys. 45, 746-762.

MacAyeal, D. R. 1992 Irregular oscillations of the West Antartic Ice Sheet. Nature 359, $29-32$.

Svendsen, B. \& Hutter, K. 1995 On the thermodynamics of a mixture of isotropic materials with constraints. Int. J. Engng Sci. 33, 2021-2054.

Thomas, I. 1941 Greek mathematical works. II. Aristarchus to Pappus of Alexandria. London: Loeb.

Vulliet, L. \& Hutter, K. 1988 Set of constitutive models for soils under slow movement. J. Geotech. Engng ASCE 114, 1022-1044. 